\title{
A DECISION ANALYIIC MODEL FOR CHEMICAL CONTROL OF SOOTY BLOTCH AND FLYSPECK DISEASES OF APPLE
}

\author{
Harvey J. Gold \\ Biomathematics Graduate Program \\ Department of Statistics, Box 8203 \\ North Carolina State University \\ Raleigh, North Carolina 27695 \\ Turner B. Sutton \\ Department of Plant Pathology, Box 7616 \\ North Carolina State University \\ Raleigh, North Carolina 27695
}

January 1985

Address correspondence to:

Dr. Harvey J. Gold

Biomathematics Graduate Program

Department of Statistics, Box 8203

NORTH CAROLINA STATE UNIVERSITY

Raleigh, NC 27695-8203 
SUMMARY

A model is presented for the optimal timing of chemical sprays to control the diseases sooty blotch and flyspeck of apple. The model accounts, at a simplified level, for dynamics of disease progress, chemical inhibition of disease spread, decay of fungistat, cost of control, and market structure for apples. Computed sensitivities and estimated uncertainties for the key parameters are used to compute Bayes optimal control decisions as well as expected value of improved accuracy. It is concluded that the decision as to chemical treatment is logically divided into two steps: (i) whether or not to treat; (ii) level of treatment. Crop quality is the most important determinant for the first step; fungistat decay rate for the second. Because of asymmetries in the economic loss curves, uncertainties lead to levels of treatment considerably above the deterministic optimum. 


\section{INTRODUCTION}

A model is presented for the optimal timing of chemical sprays to control the diseases sooty blotch and flyspeck of apple. The purpose is to provide a basis for incorporating uncertainty into control decisions, and for evaluating the relative importance of accuracy in the biological, physical and market factors.

System models have become increasingly effective tools in combating plant disease since the pioneering work of van der Plank (1963). Recent progress is reviewed in the book by Conway (1984).

Use of Bayesian decision theory in plant disease control was explored by Carlson (1970) in a study of peach brown rot. He describes procedures for assessing farmers' subjective probabilities of disease, and for combining this with forecasts of disease level based on a linear regression model. He then discusses optimal pesticide use, and compares the result with actual practice (Carlson, 1969).

In this paper, we combine the decision analytic paradigm with a mechanistic approach to modeling the system, somewhat in the spirit of Shoemaker (1982; also Shoemaker and Onstad, 1983). The overall model treats the dynamics of disease progress, chemical inhibition of disease spread, decay of the chemical control agent, cost of chemical application, and the relation between crop quality and the structure of the marketing system for apples. Each of these components is treated at a simplified level.

The optimal timing problem is split into two parts or sub-problems: Sub-problem I is that of determining the optimal timing for a given number of fungicide applications. If the dynamics of the system can be 
represented in terms of deterministic, continuously differentiable functions of time, this part of the problem may be treated using the formalism of optimal control theory, based on the maximum principle of Pontryagin (for reviews of application to pest control, see Feldman and Curry, 1982; Gold, 1983a). Given the discontinuities associated with discrete chemical application, such a formulation, al though possible, is not straightforward; it will be described in a separate report. However, the problem may be solved directly if the parameters of the system are taken to be constant at some average value over the season. In this paper, the constant parameter idealization is used, so as to simplify formulation of questions of key interest.

Sub-problem II involves accounting for the effect of disease on crop quality and the consequent effect on crop value. The diseases considered affect fruit quality, but not yield. Consideration of value loss as a function of disease incidence is complicated by the fact that apple crop value is a non-convex function of quality.

Considerable uncertainty surrounds each of the parameters for the system. Parameters which govern disease progress depend upon environmental conditions, which can only be predicted imperfectly. Value loss from a given level of disease incidence depends upon intrinsic crop quality, counted as disease free, and upon market conditions; neither of these is known at the time that control decisions must be made. With these uncertainties in mind, stress is placed on the use of the constant parameter formulation for examining questions related to the sensitivity of the optimal spray schedule to errors in the parameters, on the examination of loss curves which portray the cost of making an incorrect decision, and on the value of improved accuracy. 
The paper is patterned after the approach developed in Howard, Matheson and Miller (1977), and is organized according to the following steps :

(i) A model is developed for the system dynamics, which relates possible control inputs to the outputs. Inputs under consideration are 1 imited to number and timing of chemical applications. Outputs are possible values for disease incidence and consequent crop quality.

(ii) A model of the product market is described, which relates the output of step (i) to crop value. At the same time, costs are estimated for the control inputs.

(iii) Results of steps ( $i$ ) and ( $i$ ) are used to compute optimal number and timing of chemical applications, assuming that system parameters are known with certainty. Sensitivity to errors in the parameters is then examined.

(iv) Uncertainties in the key parameters are modeled through the development of appropriate probability distributions. These are used to estimate the best (Bayes optima1) control decisions, accounting for parameter uncertainty. The expected maximum value for removing the uncertainty (expected value of perfect information) is estimated.

(v) Finally, for the case of one of the key parameters (FM, the fraction of fresh market grade fruit), we examine the value of partial reduction of uncertainty, such as might result from a realistic estimation procedure. 


\section{DYNAMICS OF DISEASE PROGRESS}

There are seven apple diseases which occur during the summer growing period that can cause substantial loss in the southeastern United States. In this study, we investigate the control of two of these diseases, sooty blotch, caused by Gloeodes pomigena (Schw.) Colby, and flyspeck, caused by Zygophiala jamaicensis Mason [perfect stage, Schizothyrium pomi (Mont. \& Fr.) v. Arx]. These pathogens grow on fruit and twigs of apples, as well as twigs, fruit and leaves of a variety of other hosts. Usually both pathogens occur on non-sprayed fruit, and often $100 \%$ of non-sprayed fruit are affected. When either of the pathogens infects a fruit, symptoms are produced which render it unsuitable for the premium priced fresh market, though it is still suitable for manufacture of processed apple products, such as juice or sauce.

Notation is shown in Table 1.

\section{Disease Spread}

Primary inoculum for the imperfect fungus $\underline{G}$. pomigena comes from spores produced in pycnidia or from fragments of mycelial mats which overwinter on twigs within the orchard, as well as in surrounding vegetation (Baines and Gardner, 1932; Hickey, 1960). Secondary infection comes from spores and mycelial fragments from infections on woody tissues within the orchard as well as from surrounding wild hosts.

Work on $\underline{Z}$. jamaicensis is summarized by Baker, et al. (1977) and reviewed by Gold (1983b). Primary infection appears to come from ascospores discharged from perithecia which overwinter on branches and apples within the orchard and on the stem and branches of numerous wild hosts. 
Table 1. Notation

\begin{tabular}{|c|c|}
\hline B & $\begin{array}{l}\text { Market breakeven point. For } F M<B \text {, the orchard is } \\
\text { valued as if } 100 \% \text { process grade. }\end{array}$ \\
\hline$C P$ & Packing house fee. \\
\hline CS & Cost of spray treatment. \\
\hline$E_{x}(\cdot)$ & $\begin{array}{l}\text { Indicates expectation of }(\cdot) \text { with respect to probability } \\
\text { distribution for variable } \underline{x} .\end{array}$ \\
\hline$F(t)$ & Fraction of fruit diseased at time $\underline{t}$. \\
\hline FC & Fraction of "clean" fruit at harvest; $F C=1-F(T)$. \\
\hline FM & $\begin{array}{l}\text { Fraction of fresh market grade fruit at harvest, counted } \\
\text { as if free from sooty-blotch or flyspeck. }\end{array}$ \\
\hline$k(t)$ & $\begin{array}{l}\text { Inhibition parameter characterizing effectiveness of } \\
\text { fungistat at time } t \text {. }\end{array}$ \\
\hline$Q(t)$ & Concentration of fungistat in the system at time $\underline{t}$. \\
\hline$r(t)$ & Rate parameter for disease spread. \\
\hline T & Harvest time, counted from date of initial inoculation. \\
\hline VJ & $\begin{array}{l}\text { Value of process grade fruit, relative to that of large } \\
\text { tray-pack. }\end{array}$ \\
\hline VM & $\begin{array}{l}\text { Value of fresh-market grade fruit, relative to that of } \\
\text { large tray-pack. }\end{array}$ \\
\hline$x(t)$ & Ratio of infected to non-infected fruit at time $\underline{t}$. \\
\hline Y & Yield. \\
\hline$z(t)$ & Rate parameter for fungistat decay. \\
\hline
\end{tabular}


Secondary infection is caused mainly by conidia produced on primary lesions.

The logistic equation is commonly used to describe the spread of disease in a plant population. Its use in this context was introduced by van der Plank (1963), and it has been widely used in the field of plant disease epidemiology (Skylakakis, 1983; Waggoner, 1981). Letting $F(t)$ denote the fraction of fruit infected at time $t$, we have,

$$
\frac{d F}{d t}=r(t) \cdot F(t) \cdot[1-F(t)]
$$

In general, the rate parameter $\underline{r}$ must be considered to be time dependent. Among the causes of time dependence discussed by van der Plank are dependence on environmental and microenvironmental conditions, and the changing proportion of infected to infective tissue as disease progresses. Together these introduce dependence upon past environmental conditions. In the present case, there is also the complication of alternative mechanism for pathogen spread, which introduces dependence upon the history of the epidemic.

Use of equation (1) should therefore be regarded simply as an assertion that the probability that an uninfected fruit will become infected is proportional to the number of fruit already infected. The proportionality constant $\underline{r}$ is a composite of several processes, assumed to be taking place in stationary proportion. In this paper, we make the additional approximation, that the behavior over the season may be described in an average sense by taking $\underline{r}$ to be constant. Chemical Inhibition of Disease Spread

In this study, we have focused on two commonily used types of fungicides: captan and the ethylene bisdithiocarbamates (EBDC). Both 
are relatively nonspecific and exert their effects by inactivation or possibly inhibition, of SH- containing enzymes (Lyr, 1977). Except in sustained high doses, they appear to operate more as fungistats than as fungicides (Lyr, 1977; Gradis and Sutton, 1981; T. B. Sutton, unpublished data). Captan is detoxified within the cell by reaction with thiols; the cells may regenerate the needed enzymes unless exhausted. Lukens (1969) suggests that fungicidal effects result from complete exhaustion of thiols for a period of time (see Gold, 1983b for further discussion).

Drawing upon the direct mechanistic analogy with enzyme kinetics, we describe the relative degree of inhibition of disease spread by the expression,

$$
\text { inhibition }=\frac{0(t)}{k(t)+0(t)}
$$

where $Q(t)$ is concentration of fungistat in the system, and $k(t)$ is a parameter characterizing the strength of inhibition. The value of $\underline{Q}$ would normally be expressed in weight per hectare or in units of mass per unit area of foliage, depending upon the degree of detail needed.

The inhibition parameter $\underline{k}$ would in general depend upon the state of the pathogen population, as represented by its distribution among life stages. In this paper we shall, as indicated, take $\underline{k}$ to be constant.

In the presence of a fungistat operating according to equation (2), equation (1) is replaced by

$$
\begin{aligned}
\frac{d F}{d t} & =r(1-\text { inhibition }) F(1-F) \\
& =\frac{r k}{k+Q} F(1-F)
\end{aligned}
$$


For computation, it is convenient to let $X=F /(1-F)$, and to rewrite (3) as

$$
\frac{d X}{d t}=\frac{r k}{k+0} X
$$

Fungistat Residue in the System

In the apple system, the fungistat is applied in water suspension as a spray. When low concentration suspensions are used, the surfaces are normally saturated, so that the surface concentration of the residue (mass per unit area) depends primarily on the concentration of the solution.

Decay of the chemical after application depends primarily on rainfall, but also upon other factors such as temperature, wind, and light intensity (Burchfield, 1967). However, to a reasonable approximation, the average decay behavior may be described by the simple first order expression,

$$
\frac{d 0}{d t}=-z 0
$$

where $\underline{z}$ is taken to be constant. The value of $\underline{Q}$ in equation (4) may therefore be replaced by

$$
Q(t)=Q\left(t_{s}\right) e^{-z\left(t-t_{s}\right)}, \quad t \geq t_{s}
$$

where $t_{s}$ is the time of the previous spray application. The value of $Q\left(t_{s}\right)$ is the chemical residue immediately after spray application. We assume this to be the same for each application. Its value enters therefore as a constant scaling factor, and is taken to have unit value. Overall System Dynamics

Combining equation (6) with equation (4), and letting $Q\left(t_{s}\right)=1$, gives 


$$
\frac{d X}{d t}=\frac{r k}{k+e^{-z\left(t-t_{s}\right)} x}
$$

Solving this equation for a single spray time, say $t_{s}=0$ and assuming $\underline{r}, \underline{z}$, and $\underline{k}$ to be constant,

$$
x(t)=x(0)\left[\frac{k+e^{-z t}}{e^{-z t}(k+1)}\right]^{r / z}
$$

For multiple sprays, the value of $\underline{x}$ at one spray time becomes the initial value for the process until the next spray application. Letting $t_{n}$ be the date of the last spray, and $I$ be harvest time, and taking $t_{1}=0$,

$$
x(T)=x(0) \prod_{s=1}^{n-1}\left[\frac{k+e^{-z\left(t_{s+1}-t_{s}\right)}}{e^{-z\left(t_{s+1}-t_{s}\right)}(k+1)}\right]^{r / z}\left[\frac{k+e^{-z\left(T-t_{n}\right)}}{e^{-z\left(T-t_{n}\right)}(k+1)}\right]^{r / z}
$$

\section{Parameter Estimation}

The parameters $\underline{r}, \underline{z}$, and $\underline{k}$, and the initial value $F_{0}=x(0) /(1+x(0))$ from Equation (9) were fit to data reported by Hickey (1960) for control of the two diseases by captan and zineb (an EBDC fungicide). The data used consisted of 64 observations, half of which were made 5 September, and the other half on 19 September 1956. In each case, spray applications were made approximately every 2 wk, but discontinued at various times before observation. Initial time was taken as the date of earliest spray termination. It was assumed that the values of $F_{0}$ for sooty-blotch and flyspeck though different from each other did not vary from plot to plot. This gave 10 parameters to evaluate. Estimation was by a least squares procedure, using the minimization method of Powell (1964). Resulting parameter values are shown in Table 2. 
Table 2. Parameters obtained by fitting equation (9) to data of Hickey $(1966)^{(a)}$.

Disease Parameters

$$
\begin{array}{ll}
r_{S B}=0.1987 \text { days }^{-1} & F_{0, S B}=0.0000193 \\
r_{F S}=0.1213 \text { days }^{-1} & F_{0, F S}=0.00340
\end{array}
$$

\section{Fungistat Parameters}

$$
\begin{aligned}
& z_{C}=0.2736 \text { days }^{-1} \\
& z_{E}=0.1097 \text { days }^{-1}
\end{aligned}
$$

Inhibition Parameters (Mass per unit area ${ }^{(b)}$ )

$$
\begin{array}{ll}
k_{S B, C}=0.01758 & k_{S B, E}=0.05451 \\
k_{F S, C}=0.004189 & k_{F S, E}=0.004406
\end{array}
$$

Residual sum of squares $=0.1305$

Mean Square Root Residual $=0.0492$
(a) $S B=$ Sooty blotch $\quad F S=$ Flyspeck $\quad C=$ Captan $\quad E=E B D C$

(b) Relative to $O\left(t_{s}\right)$ taken to be unity. 
Further experiments were performed in 1982 and 1983 at Fletcher, North Carolina and in 1984 at Clayton, North Carolina. Captan and. mancozeb (an EBDC fungicide) were applied every 2 wh after petal fall. Sprays were discontinued on 9 or 22 July or 9 August in 1982; on 7 or 27 July or 18 August in 1983; on 3 or 14 August in 1984. In each year, the incidence and severity of sooty blotch and flyspeck were determined every 2 to 3 wk for 45 to 60 days after the last fungicide application. The apparent rate constants determined from these observations ranged from 0.088 to 0.312 for sooty blotch and from 0.065 to 0.10 for flyspeck; the fungicide decay constants ranged from 0.20 to 0.38 per day for captan and was approximately 0.11 for mancozeb.

The fits obtained were considered reasonable, given the approximations made. Moreover, examination of temperature and rainfall records appropriate to the year and Tocation for each of the sets of data shows the pattern to be plausibly related to weather pattern. Nevertheless, sensitivity calculations show the sum of squares surfaces to be very shallow, and the parameter estimates to be highly correlated. Research in progress deals with more detailed characterization of environmental dependence and will be reported separately. For the computations in this paper, we use the values shown in Table 2, unless otherwise noted. Optimum Spray Timing

If the parameters $\underline{r}, \underline{z}$, and $\underline{k}$ are taken to have constant values, expression (9) has a minimum when the spray applications are equally spaced; that is, when $t_{1}=0$ and

$$
T-t_{n}=t_{s+1}-t_{s} \text {, all } s=1, \ldots, n-1
$$


To show this, we start by assuming a single spray application. Letting $\tau$ denote time of application,

$$
x(T)=x(0) e^{r \tau}\left[\frac{k+e^{-z(T-\tau)}}{e^{-z(T-\tau)}(k+1)}\right]^{r / z}
$$

Differentiating (11) with respect to $\tau$,

$$
\frac{d x(t)}{d \tau}=x(0) \frac{1}{k+T} e^{z \tau}>0
$$

so $X(T)$ is at a minimum for $\tau=0$. That is, the lowest disease incidence occurs if the first spray is at time of initial inoculation.

For two sprays, we let $t=0$ be the time of the first spray and $\tau$ be the time of the second. We have, from equation (9),

$$
\frac{d X(t)}{d \tau}=r k X(T)\left[e^{-z(T-\tau)}-e^{-z \tau}\right]
$$

This expression is zero for $\tau=1 / 2 T$, and $i$ ts derivative with respect to $\tau$ is positive. Therefore, in any interval containing two sprays, disease incidence is lowest if the second spray is midway in the interval. By considering the spray sequence to define a sequence of overlapping two-spray intervals, it follows that minimum incidence occurs when sprays are equally spaced. 


\section{MARKET FACTORS}

Value Loss as a Function of Disease Incidence

In the typical apple market system, apples are delivered to a packing house where they are sorted and graded. They are put into one of five grade classes: large (tray pack), medium (tray pack), and small (bag) sizes for fresh market, and grades for processing into canned apple slices or processing into sauce or juice. The farmer is paid on the basis of the quantity in each grade. However, the farmer must pay the packing house for the service of grading and sorting. The amount paid for this service is generally in excess of the amount received for the processing grades. It follows that for low enough percentage of fresh market fruit, the farmer is better advised to send the entire load directly to the processor. Using the notation of Table 1, the breakeven point is $B=C P /(V M-V J)$.

Grades are primarily determined by size, color and damage (mechanical damage, insect damage and disease). The value loss that results from sooty-blotch or flyspeck is caused by shifting apples which would otherwise have been fresh market quality to process grade.

It was necessary to have a measure of value of the different grades, which would be relatively robust against market fluctuations. Such a measure was constructed, expressing the value of each grade relative to that of large tray pack apples. These relative values were determined weekly (North Carolina Department of Agriculture, 1976-1979). A $4 \mathrm{yr}$ average was determined, weighting by weekly yields from sampled commercial orchards (Proctor and Shaffer, 1983) to give an average of VM $=0.86$ for fresh market apples and $V J=0.216$ for process grade. The computational algorithm is shown in Appendix $C$. 
For this work, a relative value for $C P$ of 0.35 was used, so that $B=0.54$. To place these relative values in a real money context, a value of $\$ 52.50$ per $100 \mathrm{Kg}$ ( $\$ 10$ per 42 1b bushel) was used for large tray-pack apples.

In computing the value loss due to disease incidence, it was assumed that the diseases affect all fruit with equal probability. Cost of Chemical Application

The choice of what part of the cost of equipment operation to include will depend upon what other chemicals are being applied at the same time. Hourly cost of operating spray equipment was taken from a budget document prepared for agricultural extension personnel (Safley, Unrath and Kolbe, 1982). Time needed to spray a hectare was taken to be 37 min (15 min per acre). Variable costs (labor, fuel and repair) came to $\$ 7.81$ per ha $(\$ 3.16$ per acre); fixed costs (depreciation, insurance, $\operatorname{tax})$ were $\$ 7.88$ per ha ( $\$ 3.19$ per acre) and interest on financing was $\$ 7.29$ per ha ( $\$ 2.95$ per acre). Cost of chemical was taken to be $\$ 29.64$ per ha $(\$ 12.00$ per acre) .

Computations were performed including chemical cost only, chemical plus variable equipment costs, and including all costs. Qualitative conclusions were similar for all cost levels, and we report results including chemical and variable equipment cost. The figure of $\$ 37.45$ per ha was converted to a yield basis, assuming a yield of $23,527 \mathrm{Kg}$ per ha (500 bu per acre), to give a cost of $\$ 0.159$ per $100 \mathrm{Kg}(\$ 0.0303$ per bu) per application. 
COMPUTATIONAL RESULTS

In the following sections, results are reported separately and independently for the four disease/fungistat combinations studied. Beginning with the section on sensitivity, results on sooty-blotch are highlighted. Reasons are: simplification of the computations and of the discussion; control for sooty-blotch implies control of flyspeck as well as of other co-incident diseases, such as rots; the methodology illustrated and the qualitative nature of the conclusions are unaffected. Quantitative implications are considered in the discussion section. Optimal Treatment Levels

In computing optimal treatment levels, it was assumed that initial inoculation for both diseases occurs at petal fall. Recent studies have shown that ascospores of $\underline{Z}$. jamaicensis are mature at this time (T. B. Sutton, unpublished). Table 3 shows the optimal number of sprays for each of the four combinations of disease/fungistat, together with the calculated cost of control. Cost of control was computed assuming the same price per ha for the two chemicals. Difference in optimal number of sprays then gives an indication of the relative price premium one might be willing to pay for EBDC (current market price is about 25\% higher).

Results shown in Table 3 were obtained using the parameter values shown in Table 2. Optimal spray intervals obtained from the 1982-84 studies were approximately 10 days for sooty blotch/captan; 9 to 13 days for flyspeck/captan; approximately 21 days for sooty blotch/EDBC and 43 days for flyspeck/EBDC.

In making these computations, the unit of time was taken to be 1 day, and days were not split into fractions. When the indicated time interval 
Table 3. Optimal treatment for each disease/fungistat combination ${ }^{(a)}$.

\begin{tabular}{|c|c|c|c|c|}
\hline & $\begin{array}{l}\text { Sooty-Blotch/ } \\
\text { Captan }\end{array}$ & $\begin{array}{l}\text { Sooty-Blotch/ } \\
\text { EBDC }\end{array}$ & $\begin{array}{l}\text { Flyspeck/ } \\
\text { Captan }\end{array}$ & $\begin{array}{l}\text { Flyspeck/ } \\
\text { EBDC }\end{array}$ \\
\hline $\begin{array}{l}\text { Optimal Mo. } \\
\text { of Sprays }\end{array}$ & 8 & 5 & 7 & 3 \\
\hline $\begin{array}{l}\text { Optimal Spray } \\
\text { Intervals (Days) }\end{array}$ & 16.1 & 25.8 & 18.4 & 43 \\
\hline $\begin{array}{l}\text { Fraction of } \\
\text { Fruit Diseased }\end{array}$ & 0.0032 & 0.0045 & 0.0158 & 0.0126 \\
\hline $\begin{array}{l}\text { Value Lost }(b) \\
\text { to Disease }\end{array}$ & 0.1349 & 0.0903 & 0.147 & 0.0777 \\
\hline $\begin{array}{l}\text { Cost of } \\
\text { Trea tment }\end{array}$ & 1.270 & 0.798 & 1.113 & 0.478 \\
\hline Total Loss $(b)$ & 1.407 & 0.887 & 1.260 & 0.556 \\
\hline
\end{tabular}

(a)

Assuming F!. $=0.7, T=129$

(b) per $100 \mathrm{Kg}$. 
was not integer valued, the intervals were adjusted by rounding an appropriate proportion of the intervals up and the remaining proportion down.

\section{Loss Curves for Sub-Optimal Treatment}

In this section, cost of making sub-optimal decisions is discussed. Let $L(n)=$ value loss due to disease incidence plus cost of treatment, computed when $\underline{n}$ spray treatments are used. The cost of error is $L(n)-$ L(Optimal $n$ ). Curves are shown in Figure 1 for sooty blotch controlled by captan, for several different fractions of fresh market, FM.

Note that the optimal number of treatments is the same for all values of $F M$ above the breakeven value. In computing these curves, it was assumed that treatments are equally spaced. The extreme left-hand portion of the figure reflects the fact that no benefit derives from treatment unless it is sufficient to keep the fraction of fresh quality fruit above the breakeven level. The lower the value of $F M$, the lower the amount of damage that can be tolerated without crossing the breakeven value. The right-hand portion of the figure shows the difference between the cost of additional treatments and the additional benefit derived. The curves coincide approximately, but not precisely. The cost for overspraying is clearly small compared with the cost of underspraying. For FM below the breakeven, al1 treatment costs are lost. Sensitivity to Parameter Errors

Parameter sensitivities are presented only for the sooty blotch/ captan system. The other are qualitatively, though not quantitatively similar. 
Sensitivities are tabulated in Table 4 as the relative change needed to shift the optimal number of treatments by at least one. However, for the parameter $F M$ and for the economic parameters, no change is indicated unless the change alters the relative positions of $F M$ and the breakeven value. In the group of dynamic parameters, accuracy in the fungistat decay rate $\underline{z}$ appears to be most important.

The cost of errors in fungistat decay rate $\underline{z}$, and in the apparent rate of disease spread $\underline{r}$, are compared with that for $\underline{F M}$ in Figure 2. These curves were computed in the following manner:

Suppose that $\lambda_{0}$ is the true value of the parameter being examined, and $\lambda^{*}$ is our best guess. Let us denote by $d_{\lambda^{*}}$ the control decision based on the supposition that $\lambda^{*}$ is the true value, i.e., that $\lambda^{*}=\lambda_{0}$. Also, let $L\left(\lambda_{0}, d_{\lambda^{*}}\right)$ be the loss (cost of treatment plus value loss due to disease) when the decision is based on $\lambda^{*}$ but the true value is $\lambda_{0}$. The lowest value for $L$ would be $L\left(\lambda_{0}, d_{\lambda_{0}}\right)$, which would be attained if the estimate is correct. The excess is the cost of being wrong,

$$
C\left(\lambda_{0}, d_{\lambda^{*}}\right)=L\left(\lambda_{0}, d_{\lambda^{*}}\right)-L\left(\lambda_{0}, d_{\lambda_{0}}\right)
$$

In computing loss curves, the nominal values of Table 2 were taken as the values of $\lambda^{\star}$. Costs according to equation (13) were computed under the supposition that $\lambda_{0}=b \lambda^{*}$ where $\underline{b}$ was varied from 0.1 to 2.4 . Results are plotted in Figure 2 for parameters $\underline{F M}, \underline{r}$, and $\underline{z}$ in the sooty blotch/ captan system.

Examination of Table 4 and Figure 2 reveals the need to decompose the decision problem into a pair of hierarchically related problems. First is the decision whether to treat. Most crucial in making this decision is the ability to predict the quality of the crop relative to the breakeven 
Table 4. Relative change in parameters needed for changing optimal number of sprays for sooty-blotch/captan.

\begin{tabular}{|c|c|c|c|}
\hline \multirow[b]{2}{*}{ Dynamic Parameters } & \multirow[b]{2}{*}{ Nominal Values } & \multicolumn{2}{|c|}{ FOR CHANGE } \\
\hline & & By - 1 & By +1 \\
\hline $\begin{array}{l}r \\
z \\
k \\
F_{0}\end{array}$ & $\begin{array}{l}0.1987 \mathrm{da}^{-1} \\
0.2736 \mathrm{da}^{-1} \\
0.01753 \\
1.93 \times 10^{-5}\end{array}$ & $\begin{array}{l}-0.07 \\
-0.04 \\
-0.12 \\
-.039\end{array}$ & $\begin{array}{l}+0.22 \\
+0.11 \\
+0.38 \\
+2.3\end{array}$ \\
\hline \multicolumn{4}{|l|}{ Crop Characteristics } \\
\hline $\begin{array}{l}Y \\
\text { FM } \\
T\end{array}$ & $\begin{array}{l}23527 \mathrm{~kg} / \mathrm{ha} \\
0.70 \\
129\end{array}$ & $\begin{array}{l}-0.36 \\
-0.40(a) \\
-4.0\end{array}$ & $\begin{array}{l}+2.3 \\
+9.0(a)\end{array}$ \\
\hline \multicolumn{4}{|c|}{ Economic Parameters (relative to value of large-tray-pack) } \\
\hline $\begin{array}{l}\text { VM } \\
\text { VJ } \\
C P\end{array}$ & $\begin{array}{l}0.860 \\
0.216 \\
0.350\end{array}$ & $\begin{array}{l}-0.14^{(b)} \\
+0.5^{(b)} \\
+0.22^{(b)}\end{array}$ & +1.7 \\
\hline
\end{tabular}

(a) Absolute value change.

(b) Abrupt change to no treatment beyond these values. 
point $\underline{B}$. This is determined by parameters $\underline{F M}$, $\underline{M}$ and $\underline{C P}$. Second, if the decision is made to treat, there is the question of how many treatments to use. In this decision, accuracy in the parameter $\underline{z}$ appears to be most important, followed by parameter $\underline{r}$.

The asymmetry of the loss curve indicates that if the farmer is not certain as to the value of the parameters, he/she is well advised to err on the side of the increased chemical application, assuming that only monetary considerations are involved. This is discussed in more detail in the following section.

BAYES OPTIMAL DECISIONS AND THE VALUE OF INFORMATION Review of Underlying Concept

The conclusion of the previous section asserts that the best estimate for the parameter values should not in fact be the basis for control decisions. In order to determine the best decision, we need to combine the loss curves, such as those in Figures 1 and 2 , with estimates of the probability distributions for the parameters. In this section, we first discuss how such estimates are used, and then apply the procedure to estimates of distributions for $\underline{F M}, \underline{z}$ and $\underline{r}$.

Using the notation of the previous section and an assumed probability distribution for the true parameter, the expected cost associated with making a given decision $d_{\lambda^{*}}$ can be written,

$$
E_{\lambda} c\left(d_{\lambda^{*}}\right)=\int p(\lambda) c\left(\lambda, d_{\lambda^{*}}\right) d \lambda
$$

We choose that decision $d^{*}$ which corresponds to the lowest value for this expected cost. Because of its origin in Bayesian decision theory, this will be referred to as the Bayes optimal decision. 
The decision rule based on equation (14) is a "risk-neutral" rule, in that it accounts only for the expected, or average, gain or loss. In practice, farmers often adopt risk-averse behavior, making decisions which lead to smaller average gains, so as to avoid the risk of large occasional losses (Carlson and Main, 1976).

Because of the way in which $c\left(\lambda, d_{\lambda^{*}}\right)$ is defined, its value is zero for $\lambda=\lambda^{*}$. Therefore, if we were absolutely certain of the parameter value, the expected cost given in equation (14) would be zero. Its computed value is an estimate of the cost of uncertainty, where the uncertainty is described by the probability distribution function $p(\lambda)$. This computed value is also the cost reduction expected if the uncertainty were to be completely removed; it is therefore called the expected value of perfect information (EVPI).

of course we cannot ever hope for absolute certainty in any parameter. Moreover, the net value will be reduced by the cost of obtaining the information. The EVPI should be reaarded as an upper bound on the amount one might be willing to pay for a more accurate estimate (Howard, 1966).

In what follows, we examine the Bayes optimal decision and the EVPI for parameters FM, $\underline{z}$ and $\underline{r}$, primarily in the sooty-blotch/captan system. In each case, it is necessary to first discuss the assignment of an appropriate probability distribution. Uncertainty in Fresh Market Fraction, FM

Choice of a prior probability distribution was influenced by data presented by Shaffer, et al. (1983), based on a study of 47 sample orchard blocks in North Carolina followed over a period of 4 yr (1976-1979). From their reported frequency graph, the most appropriate distribution appears 
to be a uniform distribution with a low cut-off of close to zero, and a high cut-off slightly above 0.8. Adjusting for the reported mean incidence of sooty-blotch and flyspeck of $5.2 \%$, we choose a probability distribution which is uniform between 0.05 and 0.85 . That is, if we were to select an orchard in the North Carolina mountains at random (without knowing its unique characteristics), discounting cullage due to sootyblotch or flyspeck, we are asserting the belief that the percent fresh market fruit in any given year will almost certainly be between $5 \%$ and $85 \%$, and that any figure in that range is equally likely. This belief rests on our own informal observations, and more explicitly, on the historical data given by Shaffer, et al. (1983).

The expected value for this distribution is 0.45 . Based on this average or expected value, which is below the breakeven point, it would not be recommended that the expense of treating for these diseases be incurred. However, the computation of expected costs according to equation (14) indicates that the Bayes decision is to behave as if the fraction FM is expected to be above the breakeven point, and to apply control. The EVPI as previously defined is computed to be $\$ 0.808$ per $100 \mathrm{Kg}(\$ 0.154 /$ bu), or $\$ 189.99 / \mathrm{ha}(\$ 76.92 / \mathrm{acre})$, based on $23,527 \mathrm{~kg} / \mathrm{ha}$ (500 bu/acre).

In other words, without any information about $\underline{F M}$, other than the prior probability distribution described, we would always recommend protection. If we knew in advance the precise value of FM, we would expect to save an average of $\$ 190 /$ ha through not treating approximately $2 / 3$ of the area. This value is based on the parameter values shown in Table 2, and assuming optimal spray timing. 
Uncertainty in Pesticide Decay Rate $z$.

These computations were performed for the sooty-blotch/captan and sooty-blotch/EBDC systems. Choosing appropriate distributions for the fungistat decay constants was difficult because direct experimental data is not abundant and because the decay rate varies during the season in response to rainfal1, temperature and windspeed. Data made available to us by Harvey Reissig, Robert Seem and Bruce Stanley of the New York State Agricultural Experiment Station, Geneva, when translated into conditions appropriate for North Carolina, indicate a half-life for captan ranging from 3.5 days to 9.1 days, depending upon conditions and type of tissue. This corresponds to a decay constant of from 0.0758 to 0.1957 per day. The value estimated in Table 2 is much higher and corresponds to a half-life of 2.54 days, which is more in keeping with results reported by Smith and MacHardy (1984). A normal distribution for $\underline{z}$ with mean of $\underline{z}=0.2$ and standard deviation $\sigma$ of 0.1 seems broad enough to encompass the range of uncertainty.

In order to evaluate expression (14), a simple rectangular discrete approximation was used with the probability of the interval centered on a specific value of $z_{i}$ given by

$$
p\left(z_{i}\right)=\frac{\phi\left(\frac{z_{i}-\bar{z}}{\sigma}\right) \Delta z_{i}}{\sum_{j} \phi\left(\frac{z_{i}-\bar{z}}{\sigma}\right) \Delta z_{j}}
$$

where $\Delta z_{i}$ is the width of the interval centered on $z_{i}$ and $\phi$ is the standardized normal probability density function. Results are shown in Table 5. Also shown for comparison are results computed with the same value for $\sigma$, but with the distribution mean taken at the value shown in Table 2. 
Table 5. Effect of uncertainty in rate parameters for sooty blotch.

Captan Decay Rate

$\begin{array}{llll}\bar{z}=0.2, \sigma=0.1 & 16.1 & 12.9 & 116.1 \\ \bar{z}=0.2736, \sigma=0.1 & 21.0 & 14.3 & 126.0\end{array}$

EBDC Decay Rate

$\begin{array}{llll}\bar{z}=0.068, \sigma=.034 & 4.3 & 25.8 & 59.3 \\ \bar{z}=0.1097, \sigma=.034 & 25.8 & 21.5 & 49.4\end{array}$

Sooty Blotch Rate, $\bar{r}=0.2, \sigma=0.1$

with captan

16.1

11.7

106.2

with EBDC

25.8

16.1

108.7

\section{(a)} Assuming that treatment will be applied. 
For EBDC, the probability distribution used was based on:

(i) discussion with industry representatives; $(i i)$ the value indicated in Table 2; and (iii) leaf measurements at an experimental orchard in Clayton, N. C. during the summer of 1984 (T. B. Sutton, unpublished). A normal distribution for the decay constant was chosen, with mean 0.068 and standard deviation of 0.034 . Results of the computation are shown in Table 5, and compared with computations using the same standard deviation, but mean value shown in Table 2 .

It is interesting to note that while the deterministic optimal spray interval in both cases is strongly dependent on the assumed mean, the Bayes optimal interval and the EVPI are reasonably independent of the assumed mean, and are presumably dominated by the uncertainty factor. Uncertainty in Disease Spread Rate Parameter $r$.

Also shown in Table 5 are results of computations related to uncertainty in the rate parameterfor sooty-blotch as controlled by captan or by EBDC. A normal distribution was assumed, with mean of 0.2 and standard deviation of 0.1 . The cost of such uncertainty, as reflected in the EVPI, is about the same for the two fungistats.

\section{EXPECTED VALUE OF PARTIAL INFORMATION}

Although absolute certainty may not be possible, we can often reduce uncertainty through research efforts, field observations, and construction of models which relate observations to parameter values. These efforts are often costly in terms of time, effort and physical resources. The EVPI gives us the maximum expected return for such efforts, and logically imposes an upper limit on the amount of effort that would be justifiable. 
In this section, we take into account that the effort will lead to reduced but not eliminated uncertainty. We examine the question with respect to the parameter FM only. The other two parameter types which we have been discussing (the rate constants for disease spread and for fungistat decay) are more appropriately treated as time functions, and will be further examined in a later report.

Information about FM might come from several sources. One possibility is the examination of the detailed records of an individual orchard and the expectation of year-to-year correlation with respect to fruit quality. Another possibility is through construction of a fruit development model, which relates early season measurements in the orchard to projections about likely fruit quality. We are currently examining both of these possibilities. Such information is combined with the prior distribution discussed previously to give us a revised probability distribution, with the effect of reducing the expected loss as given by equation (14). The amount of reduction is the expected value of the information (EVI). Perfect information of course reduces the expected loss in equation (14) to zero.

The computation scheme starts with the idea that the result of any estimation procedure will depend upon the true value of the parameter being estimated, but will be subject to some error distribution.

Suppose we knew the true value of $F M$ to be $F M_{0}$, and had to guess in advance the outcome of an estimation or measurement procedure. Since the procedure is less than completely accurate, the best we could do is to assign it a probability distribution, which we designate as $f\left(x \mid F M_{0}\right)$, the probability distribution, for the measurement $\underline{x}$, given the true value of $F M_{0}$. This distribution has a shape and spread which depends upon the types of error associated with the measurement procedure. 
of course, our real interest is in the orobability distribution $\xi(F M \mid x)$, the probability distribution on $\underline{F M}$, given knowledge of $\underline{x}$. We can relate this distribution to the previous expression by means of Bayes' formula (see, for example, De Groot, 1970),

$$
\xi(F M \mid x)=\frac{f(x \mid F M) \xi(F M)}{f(x)}
$$

where $\xi(F M)$ is the prior distribution on $F M$ (that is, the distribution we would have used, were the estimate $\underline{x}$ not available), and $f(x)$ is the marginal distribution on $\underline{x}$,

$$
f(x)=\int f(x \mid F M) \xi(F M) d F M
$$

Our purpose in this section is to examine the value of a potential estimate of $F M$ as a function of its accuracy. This is made more convenient if we can choose a suitable parametric family of probability distributions, and then express the accuracy as a function of the distribution parameters. In choosing an appropriate family, we note that the estimate is to be obtained by some method of projection, rather than population sampling, so that the usual reasoning based on statistical sampling distributions is not applicable. Nevertheless, given that we are estimating a proportion, the standard beta distribution between zero and one seems to be appropriate (De Groot, 1970). Use of the beta distribution, however, requires numerical evaluation of several difficult integrals. We therefore choose to approximate the distribution of such an estimate by a normally distributed variable, with the conditions,

$$
E(X \mid F M)=F M
$$

We then compute the EVI for the estimate as a function of the standard deviation, $\sigma$. 
The approximation (18) will be expected to break down as $\sigma$ becomes large so that $\underline{x}$ has substantial density outside the interval $[0,1]$.

The decision rule for crop treatment will be to treat the crop if the estimate $\underline{x}$ is above some value $x_{B}$ and not otherwise. For a given $x_{B}$ and a given estimate $\underline{x}$, let the value per $100 \mathrm{~kg}$ of the crop be designated as $V\left(x, x_{B}\right)$. For $x<x_{B}$, the decision is not to treat, so $V\left(x, x_{B}\right)=V J$, the value of process fruit. For $\underline{x}$ greater than $x_{B}$, $V\left(x, x_{B}\right)$ will have a value which will depend upon $F M$. Its expectation must therefore be taken with respect to the probability distribution for $\underline{F M}$, given the observation $\underline{x}$,

$$
\begin{aligned}
E_{F M \mid x} V\left(x, x_{B}\right)= & (V J-C S) \\
& +F C(V M-V J) \int_{B / F C}^{0.85} F M \xi(F M \mid x) d F M \\
& -C P \int_{B / F C}^{0.85} \xi(F M \mid x) d F M .
\end{aligned}
$$

The first term here accounts for the value of the fruit if it were shinped directly to the processor, reduced by the amount spent on treatment. The second term is the expected additional value, which will be received only if the fruit is shipped to the fresh market. Since FM is the fraction of fresh market fruit, counted as completely free of the disease, the actual fraction of fresh market fruit is reduced by the fraction of disease. Letting $F C$ be the fraction of fruit free of disease at harvest, the crop goes to the packing house only if $F M>B / F C$. The last term is the packing house cost, which is incurred only if FM > B/FC. 
The best (Bayes optimal) value to use for $x_{B}$ is the value for which the expression in equation (19) equals VJ, the value that would obtain without treatment. Details related to the computation of $x_{B}$ are shown in Appendix A. Results are shown in the second column of Table 6.

Before we go to the effort of making the estimate, we do not know what its value will be. In order to judge if the effort is likely to be worthwhile, the expected value of the information is computed by averaging the possible values for $E_{F M \mid x} V\left(x, x_{B}\right)$ with respect to the probability distribution over $\underline{x}$, as given by equation (17). Details related to the computation are shown in Appendix B. Results are shown in the last two columns of Table 6 .

\section{DISCUSSION AND CONCLUDING REMARKS}

The representation of the system dynamics which we have used is, as has been pointed out, at a simplified level. Moreover, the losses and optima shown were computed for the individual diseases independently. The approach used, however, is useful for the purpose of developing the decision methodology, and for investigating the value of improved information (i.e., reduced uncertainty) concerning key parameters. This is substantiated by the agreement of the computed optima shown in Tables 3 and 5 with empirically arrived at recommendations.

It should also be noted that in the sensitivity computations, we varied only one parameter at a time. Greater detail would involve replacing the curves of Figure 1 and Figure 2 with appropriate loss surfaces. 
Table 6. Bayes optimal treatment decision at different accuracies for the estimate of FM.

\begin{tabular}{lccc}
\hline $\begin{array}{l}\text { STD. DEV. } \\
\text { of } \\
\text { ESTIMATE }\end{array}$ & $\begin{array}{l}\text { DECISION } \\
\text { BREAKPOINT } \\
\mathrm{x}_{\mathrm{B}}\end{array}$ & $\begin{array}{l}\text { EVI/100Kg } \\
\text { (dol1ars) }\end{array}$ & $\begin{array}{l}\text { EVI/HA (a) } \\
\text { (do11ars) }\end{array}$ \\
\hline 0 (perfect info.) & 0.583 & .808 & 189.99 \\
0.05 & 0.574 & .761 & 179.08 \\
0.1 & 0.542 & .646 & 151.91 \\
0.2 & 0.467 & .278 & 65.46 \\
0.25 & 0.431 & .121 & 28.47 \\
\hline
\end{tabular}

(a) Assuming $23,527 \mathrm{Kg} / \mathrm{ha}$ ( $500 \mathrm{bu} / \mathrm{acre})$. 
Similarly, the value-of-information computations of Tables 5 and 6 are based on allowing only one parameter at a time to be uncertain. The effects of joint uncertainties in several variables will in general not be additive.

Several conclusions seem to emerge. First is that the decision as to chemical treatment for these diseases is logically divided into two steps: (1) whether or not to treat; (2) the level of treatment, if decision is made to treat. Note that the decision breakpoint in this case is based on projections as to the quality of fruit. It is not the same as the economic threshold, which is based on the pest level and projections as to pest related reduction in yield (Stern, et a1., 1959; Headley, 1972).

Computations in the subsection on Uncertainty in FM indicate that the Bayes optimal decision in the absence of further specific information, would lead to the treatment of large amounts of acreage for sooty blotch and flyspeck, which do not in any case qualify for the fresh market. This conclusion would be even stronger if risk averse behavior were accounted for, as indicated in the discussion following equation (14). The result is based on the overall uniform probability distribution suggested by the plots in Shaffer, et al. (1983). Of course, an individual farmer may be in a position to make a better guess based on his knowledge of his own orchard and its history. The results in Table 6 indicate that even relatively crude estimates of $F M$ may have considerable value. Examination of individual orchard records would allow determination of the extent to which historical information concerning the success of a given orchard is already being used in making selective decisions. 
The question of whether FM exceeds the breakeven point for treatment will also depend upon the cost of treatment, and upon the parameters VM, $\underline{\text { V }}$ and $\underline{C P}$, the values for fresh market and for process fruit and the packing house fee. These will depend upon the general state of the economy, the total supply of apples, and the supply of other fruits which may compete with apples. None of these is known in advance with , certainty. Table 6 may therefore be interpreted as indicating the potential value of economic forecasts. A more complete economic treatment would also include such factors as costs of finance and opportunity cost. Allowance would also have to be made for the possibility that even if the projected value of $F M$ is below the decision breakpoint, fungicides would have to be applied to protect against other diseases, in particular, the rots.

Given a decision to apply chemical control, the best treatment schedule is a series of equally spaced applications, assuming that the dynamic parameters can be taken as constant. As shown in Table 4 , the fungistat decay rate $\underline{z}$, and the apparent rate parameter for disease spread $\underline{r}$, are the most important for this decision. Table 5 shows the EVPI values. As we have already stated, these figures are based on the varying of only one parameter at a time.

In addition to the possibility that needless treatment could be avoided by better projections as to crop quality, the values reported in Tables 5 and 6 , together with the loss curves in Figures 1 and 2, demonstrate that uncertainties in the system parameters increase the recommended level of treatment. This is true even without accounting for risk-averseness; it results from the lack of symmetry of the loss curves. That is, underspraying by two or more sprays leads to a much greater loss than overspraying 
by the same amount. In the face of uncertainty, therefore, a lower expected loss results from overspraying. However, the loss curves are more nearly symmetric near the minimum point. Therefore, information which is good enough to concentrate the probability distributions into fairly narrow bands would lead to Bayes optimal decisions close or identical to the deterministic optimum. For this, the information would have to be good, but not perfect.

The effect of taking into account simultaneous effects of sooty blotch and flyspeck, and of including other diseases would, we believe, increase the difference between the Bayes and the deterministic optima. This is because losses from underspraying would be increased, while losses from overspraying would be unaffected. This would increase the asymmetry of the loss curves.

Our discussion on the value of information is apart from the cost of obtaining it. We expect such costs to consist of two parts. One is the research cost, including development of models which would allow more accurate estimation of the system parameters; second is the cost of using the models, including the cost of monitoring the orchards and providing the necessary season-specific observational data. 


\section{APPENDIX A \\ Estimation of $x_{B}$}

It will be convenient to use the notation $\Phi\left(\frac{a-x}{\sigma}\right)$ to denote the cumulative up to $\underline{a}$ of the normal distribution with mean $\underline{x}$ and variance $\sigma$, and to let

$$
\Delta \Phi(a, b, x, \sigma)=\Phi\left(\frac{b-x}{\sigma}\right)-\Phi\left(\frac{a-x}{\sigma}\right)
$$

be the integral of such a distribution over the interval $[a, b], a \leq b$.

In order to evaluate the second term of the r.h.s. of equation (18), we note that $\xi(F M \mid x)$ is given by equation (15), where $f(x \mid F M) \sim N\left(F M, \sigma^{2}\right), \xi(F M) \sim$ $u[0.05,0.85]$, and $f(x)$, given by (16) may therefore be written,

$$
f(x)=\Delta \Phi(0.05,0.85, x, \sigma) /[0.85-0.05]
$$

The term becomes

$$
\frac{0.8}{\sigma \sqrt{2 \pi} f(x)} F C(V M-V J) \int_{B / F C}^{0.85} e^{-(x-F M)^{2} / 2 \sigma^{2}} d F M .
$$

This integral is, to a normalizing factor of $\Delta \Phi(B / F C, 0.85, x, \sigma)$, the mean of the distribution $N\left(x, \sigma^{2}\right)$, truncated to the interval [B/FC, 0.85].

Johnson and Kotz (1970) give the mean of a variable $N\left(x, \sigma^{2}\right)$ truncated to the interval $[A, B]$ as

$$
E_{[A, B]} N\left(x, \sigma^{2}\right)=x+\left[Z\left(\frac{A-x}{\sigma}\right)-Z\left(\frac{B-x}{\sigma}\right)\right] / \Delta \Phi(A, B, x, \sigma)
$$

Substituting $(A-1)$ and $(A-3)$ into $(A-2)$ gives

$\operatorname{Term} 2(X)=\frac{\Delta \Phi(B / F C, 0.85, x, \sigma)}{\Delta \Phi(0.05,0.85, x, \sigma)} F C(V M-V J) \cdot E_{[B / F C, 0.85]}(F M \mid X)$ 
The last term of equation (19) is simply the density given by (16) integrated over the interval $[B / F C, 0.85]$, and is given by

$$
\operatorname{Term} 3(x)=C P \frac{\Delta \Phi(B / F C, 0.85, x, \sigma)}{\Delta \Phi(0.05,0.85, x, \sigma)}
$$

The value of $x_{B}$ is then arrived at as the zero of the expression,

$$
\text { CS }+\operatorname{Term} 2(X)-\operatorname{Term} 3(X) \text {. }
$$

Evaluation was performed in Fortran using the error function to evaluate \$, and the IMSL routine ZREAL2 (a Newton method) to svaluate the zero. 
APPENDIX B

Expected Value of Information

The expected value $E_{F M \mid x} V\left(x, x_{B}\right)$ is integrated with respect to the probability distribution over $\underline{x}$. Since $V\left(x, x_{B}\right) \equiv V J$ for $x<x_{B}$,

$$
E_{x} V\left(x, x_{B}\right)=V J-C S \int_{x_{B}}^{1} f(x) d x+F C(V M-V J) \int_{x_{B}}^{1} \int_{B / F C}^{0.85} F M f(x \mid F M) \xi(F M) d F M d x-
$$$$
-C P \int_{x_{B}}^{1} \int_{B / F C}^{0.85} f(x \mid F M) \xi(F M) d F M d x
$$

The first term is the "base" value of process grade fruit. The second is the cost of spraying, incurred only if $x>x_{B}$. Third is the expected excess value of fruit delivered to the packing housing, incurred only if $x>x_{B}$, and $F M>B / F C$. The final term is the packing house fee, incurred under the same conditions.

To evaluate the second term, $f(x)$ is expressed as in $(A-1)$, resulting in two integrals, each of which may be integrated by parts.

Similarly, the integral in term four may be expressed as

$$
\frac{1}{0.8} \int_{x_{B}}^{1} \Delta \Phi(B / F C, 0.85, x, \sigma) d x
$$

resulting in two integrals, each of which may be integrated by parts.

The integral in term three is somewhat more difficult. Integration by parts in this case leads to a term which is proportional to

$$
E_{[B / F C, 0.85]}\left(F M^{2} \mid X\right) \text {. }
$$


This is expressed in terms of the variance and square of the expectation, and use is made of the expression given by Johnson and Kotz for the variance of a truncated normal. The result is,

$\int_{x_{B}}^{1} \int_{B / F C}^{0.85} F M f(x \mid F M) \xi(F M) d F M d x=\frac{1}{0.8}\left\{\frac{1}{2} \Phi\left(\frac{-0.15}{\sigma}\right)-\frac{1}{2} x_{B}^{2} \Phi\left(\frac{0.85-x_{B}}{\sigma}\right)\right.$

$$
\begin{aligned}
& +\frac{\Delta \Phi\left(x_{B}, 1,0.85, \sigma\right)}{2}\left[\operatorname{VAR}_{\left[x_{B, 1}\right]} N\left(0.85, \sigma^{2}\right)+E_{\left[x_{B, 1}\right]}^{2} N\left(0.85, \sigma^{2}\right)\right] \\
& -\frac{1}{2} \Phi\left(\frac{B / F C-1}{\sigma}\right)+\frac{1}{2} x_{B}^{2} \Phi\left(\frac{\left.B / F C-x_{B}\right)}{\sigma}\right) \\
& -\frac{\Delta \Phi\left(x_{B}, 1, B / F C, \sigma\right)}{2}\left[\operatorname{VAR}_{\left[x_{B, 1}\right]} N\left(B / F C, \sigma^{2}\right)+E_{\left[x_{B, 1}\right]}^{2} N\left(B / F C, \sigma^{2}\right)\right] \\
& \left.-\sigma^{2} \Delta \Phi\left(x_{b}, 1,0.85, \sigma\right)+\sigma^{2} \Delta \Phi\left(x_{B}, 1, B / F C, \sigma\right)\right]
\end{aligned}
$$

where $\operatorname{VAR}_{[A, B]} N\left(x, \sigma^{2}\right)$ indicates the variance of a distribution $N\left(x, \sigma^{2}\right)$ truncated to $[A, B]$.

Computation is straightforward, using the Fortran error function to compute terms in $\Phi\left(\frac{a-b}{\sigma}\right)$ and the expression given for variance by Johnson and Kotz, 


$$
\begin{aligned}
\operatorname{VAR}_{[A, B]} N\left(x, \sigma^{2}\right)= & \left\{1+\frac{\left(\frac{A-x}{\sigma}\right) \underline{Z}\left(\frac{A-x}{\sigma}\right)-\left(\frac{B-x}{\sigma}\right) Z\left(\frac{B-x}{\sigma}\right)}{\Delta \Phi(A, B, x, \sigma)}\right. \\
& {\left.\left[\frac{Z\left(\frac{A-x}{\sigma}\right)-Z\left(\frac{B-x}{\sigma}\right)}{\Delta \Phi(A, B, x, \sigma)}\right]^{2}\right\} \sigma^{2} }
\end{aligned}
$$




\section{APPENDIX C \\ Computation of Relative Grade Values}

Prices are quoted in the market report on a per-bushel basis.

Let

$$
\begin{aligned}
& y_{s, t}=\text { yield in bushels from sample tree } \underline{s} \text { in wk } \underline{t} . \\
& m_{s, c}=\text { wt of harvest from tree } \underline{s} \text { in grade class } \underline{c} . \\
& m_{s}=\text { total wt of harvest from tree } \underline{s} . \\
& w_{c, t}=\text { weighting factor for class } \underline{c} \text { and wk } \underline{t} . \\
& p_{c, t}=\text { price quoted (per bushel) for class } \underline{c} \text { in wk } \underline{t} . \\
& p_{1, t}=\text { price quoted for large-tray-pack apples in wk } \underline{t} . \\
& v_{c}=\text { relative value of class } \underline{c} .
\end{aligned}
$$

Then,

$$
\begin{aligned}
& w_{c, t}=\sum_{s} y_{s, t}\left(m_{s, c} / m_{s}\right) \\
& v_{c}=\frac{\sum_{t} w_{c, t}\left(p_{c, t} / p_{1, t}\right)}{\sum_{t} w_{c, t}}
\end{aligned}
$$

For purposes of this report, $\underline{V M}$ and $\underline{V}$ were taken as simple averages over the three fresh-market grades and two process grades, respectively. 


\section{ACKNOWLEDGMENTS}

We should like to thank Harvey Reissig, Robert Seem and Bruce Stanley of the New York Experiment Station at Geneva for sharing with us their results on pesticide decay. We thank Charles Safley for assistance in use of the North Carolina Extension Service budget document 1981-82, Gerald Carlson and Richard Unrath for discussion concerning market operation, Phillip Shaffer for discussions concerning fungicide decay, and H. R. van der Vaart and John Monahan for advice concerning the integral evaluations. We particularly appreciate suggestions made by Dr. Carlson after review of a draft of this manuscript.

Use of trade names does not constitute endorsement by the North Carol ina Agricultural Research Service of the products named, and does not imply criticism of similar ones not mentioned.

This work was supported in part by the U. S. Department of Agriculture under Agreement No. 82-CRSR-2-1000.

Paper 0000 of the Journal Series of the North Carolina Agricultural Research Service, Raleigh, N. C. 27695-7610. 


\section{REFERENCES}

Baines, R. C. and M. W. Gardner. (1932). "Pathogenicity and Cultural Characteristics of the Apple Sooty Blotch Fungus," Phytopathology 22: $937-952$.

Baker, K. F., L. H. Davis, R. D. Durbin, and W. C. Snyder. (1977). "Greasy Blotch of Carnation and Flyspeck of Apple: Diseases Caused by Zygophiala jamaicensis," Phytopathology 67:580-588.

Burchfield, H. P. (1967). "Chemical and Physical Interactions," in Fungicides, An Advanced Treatise, Editor D. C. Torgeson, Volume I: 463-508, Academic Press, New York.

Carlson, G. A. (1969). "Bayesian Analysis of Pesticide l'se," Proc.

Amer. Stat. Assoc., Section on Business and Economic Statistics, pp. 411-415.

Carlson, G. A. (1970). "A Decision Theoretic Approach to Crop Disease Prediction and Control," American J. of Agricul tural Economics 52: 216-223.

Carlson, G. A. and C. E. Main. (1976). "Economics of Disease-Loss Management," Annual Review of Phytopathology 14:381-403.

Conway, G. R. (Editor)(1984). Pest and Pathogen Control: Strategic, Tactical, and Policy Models. John Wiley \& Sons, Inc., New York. 488 pp. De Groot, M. H. (1970). Optimal Statistical Decisions. McGraw-Hill, New York. 489 pp.

Feldman, R. M. and G. L. Curry. (1982). "Operations Research for Agricultural Pest Management," Operations Research 30:601-618. Gold, H. J. (1983a). A Survey of Decision Modeling Formalism as Applied to Pest Management, Institute of Statistics Mimeo Series, 
No. 1634, Biomathematics Series No. 15, North Carol ina State University, Raleigh, NC. 19 pages (available on request).

Gold, H. J. (1983b). Framework for Modeling the Epidemiology of Sooty Blotch and Flyspeck Diseases for Apple and Their Control. Institute of Statistics Mimeo Series No. 1635, Biomathematics Series No. 16, North Carolina State University, Raleigh, NC. 21 pages. (available upon request).

Gold, H. J., P. L. Shaffer, M. Wann, and E. Johnson. (1983). "A Modeling Strategy for the Orchard Ecosystem," in Integrated Pest and Orchard Management Systems for Apples in North Carolina, Editors G. C. Rock and L. J. Apple. Pages 161-172, Technical Bulletin No. 276, North Carolina Agricultural Research Service, Raleigh, NC.

Gradis, W. H. and T. B. Sutton. (1981). "Effect of Insecticides, Nutrients, and Adjuvants on In Vitro Fungistatic and Fungicidal Activity in Captan and Mancozeb," Plant Disease 65:357-358.

Headley, J. C. (1972). "Defining the Economic Threshold," in Pest Control Strategies for the Future, Pages 100-108, National Academy of Science, Washington, D. C.

Hickey, K. D. (1960). The Sooty Blotch and Flyspeck Diseases of Apples, with Emphasis on Variation within Gioeodes Pomigena (Schw.) Colby. Ph.D. Thesis, Department of Botany and Plant Pathology, Penn State University, College Station, Pennsylvania. 127 pages.

Howard, R. A. (1966). "Information Value Theory," IEEE Trans. Systems Science and Cybernetics SSC-2: $22-26$.

Howard, R. A., J. E. Matheson and K. L. Miller (Editors). (1977).

Readings in Decision Analysis, Decision Analysis Group, SRI, International, Menlo Park, CA. 611 pages. 
Johnson, N. L. and S. Kotz. (1970). Continuous Univariate

Distributions, Volume 2. Houghton-Mif1in, Boston, MA . 306 pages. Lukens, R. J. (1969). "Heterocyclic Nitrogen Compounds," in Pesticides: An Advanced Treatise, Volume II, Editor D. C. Torgeson, Pages 395445. Academic Press, New York.

Lyr, H. (1977). "Mechanism of Action of Fungicides," in Plant Disease, An Advanced Treatise, Volume I, Editors J. G. Horsfall and E. C. Cowling, pages 239-261. Academic Press, New York.

North Carolina Department of Agriculture and United States Department of Agricul ture (1976-1979), Marketing Western North Carolina Apples and Vegetables, Federal State News Service.

Powe11, M. J. D. (1964). "An Efficient Method for Finding the Minimum of a Function of Several Variables without Calculating Derivatives," Computer Journal 7:155-162.

Proctor, C. H. and P. L. Shaffer. (1983). "Orchard and Tree Sample Selection and Data Analysis," in Integrated Pest and Orchard Management Systems for Apples in North Carolina, Editors G. C. Rock and J. L. Apple, pages 7-10, Technical Bulletin No. 276, North Carolina Agricultural Reserch Service, Raleigh, NC.

Safley, C., C. R. Unrath and M. H. Kolbe. (1982). "Fresh Apples: Estimated Revenue, Operating Expenses, Annual Ownership Expenses and Net Revenue per Acre: Budget, 1981-82," North Carol ina Extension Service, Raleigh, NC.

Shaffer, P. L., C. R. Unrath, T. B. Sutton, and G. C. Rock. (1983). "Fruit Quality Based on Grade Standards," in Integrated Pest and Orchard Management Systems for Apples in North Carolina, Editors 
G. C. Rock and J. L. Apple, pages 57-66, Technical Bulletin No. 276, North Carolina State University, Raleigh, NC.

Shoemaker, C. A. (1982). "Optimal Integrated Control of Univoltine Pest Populations with Age Structure," Operations Research 30:40-61. Shoemaker, C. A. and D. W. Onstad. (1983). "Optimization Analysis of the Integration of Biological, Cultural, and Chemical Control of Alfalfa Weevil," Environmental Entomology 12:286-295. Skylakakis, G. (1983). "Theory and Strategy of Chemical Control," Annual Review of Phytopathology 21:117-135. Smith, F. D. and W. E. MacHardy. (1984). "The Retention and Redistribution of Captan on Apple Foliage," Phytopathology 74:894-899.

Stern, V. M., R. F. Smith, R. van den Bosch and K. S. Hagen. (1959). "The Integrated Control Concept," Hilgardia 29:81-95. van der Plank, J. E. (1963). Plant Diseases: Epidemics and Control. Academic Press, New York. 349 pages. Waggoner, P. E. (1981). "Models of Plant Disease," BioScience 31:315-319. 


\section{FIGURE CAPTIONS}

Figure 1. Loss function, using incorrect number of spray applications, at different values for FM.

Figure 2. Cost of error in parameters $\underline{z}, \underline{r}$, and $\underline{F M}$, shown as cost of basing decision on estimate $\lambda^{*}$ when $\lambda_{0}=b \lambda^{*}$ is true value. 


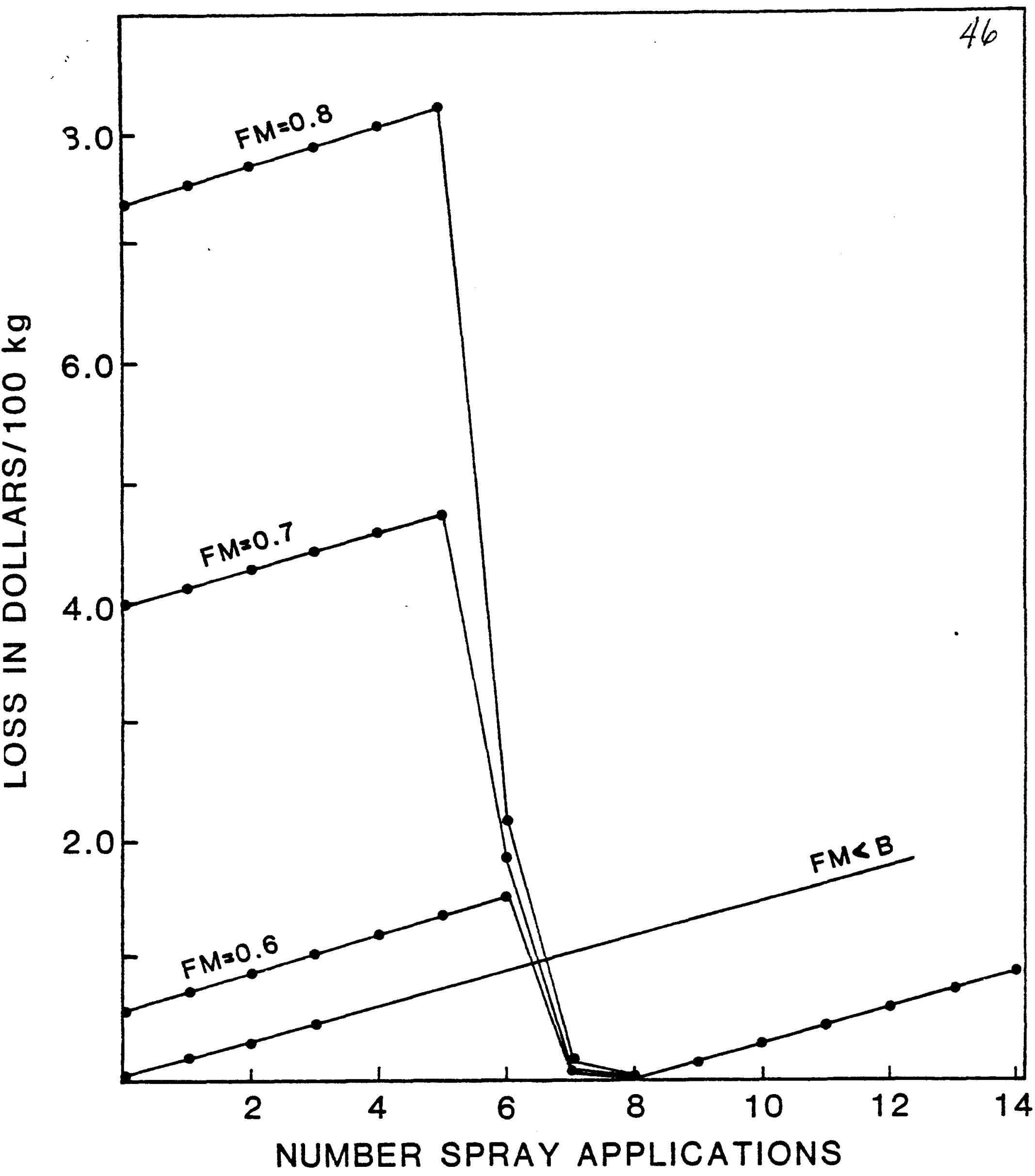




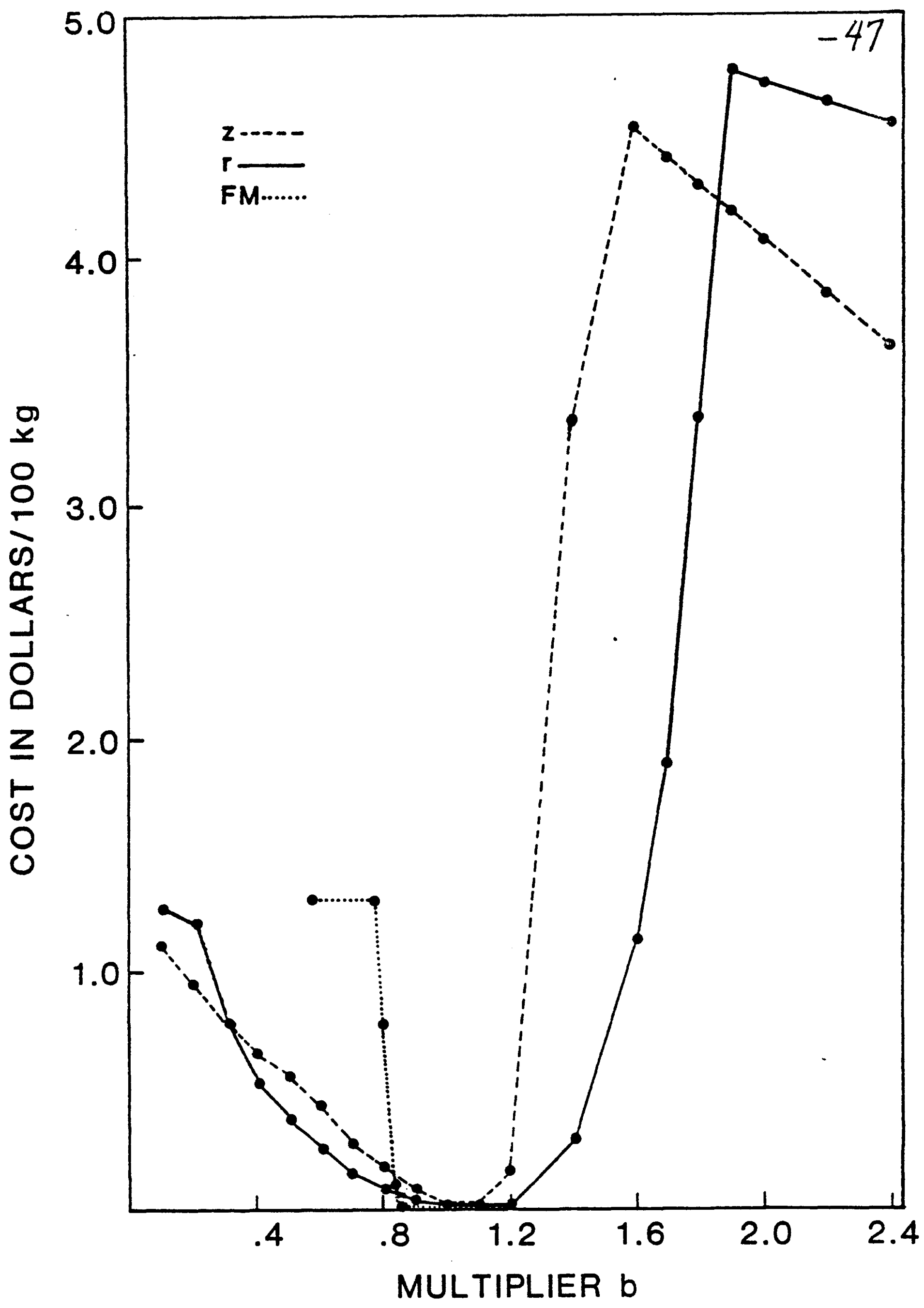

